\title{
Adaptive Vorsteuerung für Windenergieanlagen
}

\author{
Adaptive Feed Forward Control for Wind Turbines
}

\author{
David Schlipf*, Po Wen Cheng, Stiftungslehrstuhl Windenergie am Institut für Flugzeugbau, Stuttgart \\ * Korrespondenzautor: schlipf@ifb.uni-stuttgart.de
}

Zusammenfassung Der Beitrag beschreibt, wie Windmessungen mit LIDAR in einer Vorsteuerung verwendet werden können, um die Drehzahlschwankungen und damit die Belastungen von Windenergieanlagen zu reduzieren. Kernstück dieser Vorsteuerung ist ein Filter, der adaptiv auf die aktuellen Messungen eingestellt werden muss, da sich die Prädiktionszeit und die Korrelation zwischen Vorhersage und Anlagenverhalten kontinuierlich ändern. Die Ergebnisse werden mit Mess- daten einer $5 \mathrm{MW}$ Anlage validiert. $\leadsto$ Summary This paper presents how LIDAR measurements can be used in a feed forward control to reduce rotor speed variation and loads on wind turbines. Core of this control strategy is an adaptive filter that takes into account the continuous changes in the prediction time and in the correlation between the turbine reaction and the preview. Results will be validated with measurement data of a $5 \mathrm{MW}$ wind turbine.

Schlagwörter Vorsteuerung, LIDAR basierte Regelung, Lastreduktion $\leadsto \quad$ Keywords Feed forward control, LIDAR assisted control, load reduction

\section{Einleitung}

Die Aufgaben eines Reglers können in Sollwertnachführung und Störgrößenunterdrückung eingeteilt werden [1]. Die Regelung von Windenergieanlagen beschränkt sich hauptsächlich auf letztere: Die Anlage muss auf Änderungen in Windgeschwindigkeit, Windrichtung und Scherungen reagieren. Dies legt nahe, Informationen über das einströmende Windfeld in die Regelung zu integrieren. In den letzten Jahren wurde durch die Entwicklung der LIDAR ("LIght Detection And Ranging") Technologie in diesem Bereich vermehrt geforscht. Neben dem Einsatz bei der zyklischen Blattverstellung [2], Drehmomentregelung und Windnachführung [3] sind vor allem die Vorteile bei der kollektiven Blattverstellung vielversprechend [4-6].

Diese Arbeit zeigt, dass die Vorsteuerung des kollektiven Blattwinkels zur Minimierung der Drehzahlvariation in ein regelungstechnisches und in ein messtechnisches Problem unterteilt werden kann. Diese Trennung führte $\mathrm{zu}$ erfolgreichen Feldtests [7;8]. Das regelungstechnische Problem kann durch eine einfache nichtlineare Vorsteuerung zufriedenstellend gelöst werden, wenn die rotoreffektive Windgeschwindigkeit bekannt ist. Eine Untersuchung realer Messdaten eines LIDAR Systems und eines Ultraschallanemometers verdeutlicht, dass die Herausforderung darin liegt, die rotoreffektive Windgeschwindigkeit möglichst genau und rechtzeitig zu messen. Durch die Erfassung der Korrelation zwischen Windmessungen und Reaktion einer Windenergieanlage kann gezeigt werden, dass nur niedere Frequenzen der Störgröße gemessen und damit kompensiert werden können. Um negative Effekte durch nicht korrelierte Frequenzen $\mathrm{zu}$ vermeiden, wird ein Filter benötigt, der wiederum eine Messung vor der Anlage bedingt, um den Zeitversatz durch die Filterung zu kompensieren. Da sich die Korrelation und die Zeit zwischen Messung und Einwirken auf die Anlage kontinuierlich ändern, muss der Filter adaptiv entworfen werden. Kann dann die Wind- 
geschwindigkeit verlässlich vorhergesagt werden, so ist diese Information sowohl in das Optimierungsproblem eines modellprädiktiven Reglers [9], aber auch in eine Vorsteuerung integrierbar.

Am Anfang dieser Arbeit wird das verwendete Modell einer Windenergieanlage beschrieben, die Vorsteuerung entworfen und unter der Annahme von perfekter Messung getestet. Anschließend werden Windmessungen daraufhin untersucht, ob sie die benötigte Information für die Vorsteuerung erfassen können. Damit wird die Vorsteuerung um einen adaptiven Filter erweitert. Abschließende Simulationen auf Basis der Messdaten zeigen signifikante Lastreduktionen.

\section{Modellierung einer Windenergieanlage}

Für den Entwurf der Vorsteuerung, die Schätzung der rotoreffektiven Windgeschwindigkeit, aber auch für die Simulationen in Kapitel 7 wird ein reduziertes, nichtlineares Modell einer Windenergieanlage verwendet (siehe Bild 1). Dabei werden basierend auf [9] nur die erste Turmbiegemode, die Rotordrehung und die Blattverstellung modelliert:

$J \dot{\Omega}+M_{g} / i=M_{a}$

$m_{T} \ddot{x}_{T}+c_{T} \dot{x}_{T}+k_{T} x_{T}=F_{a}$

$\ddot{\theta}+2 \xi \omega \dot{\theta}+\omega^{2}\left(\theta-\theta_{c}\right)=0$.

Der Drallsatz (1a) für die Rotordrehzahl $\Omega$ repräsentiert die Umwandlung des aerodynamischen Moments $M_{a}$ in ein elektrisches Generatormoment $M_{g}$. Die Konstanten $i$ und $J$ sind die Getriebeübersetzung und die gesamte Trägheit des Rotors und des Generators. Gleichung (1b) beschreibt die Dynamik der Turmkopfauslenkung $x_{T}$, angeregt durch den Schub $F_{a}$ in Windrichtung. Hierbei sind $m_{T}, c_{T}$, und $k_{T}$ die äquivalente Masse, Dämpfung und Steifigkeit. Die Verstellung des kollektiven Blattwinkels $\theta$ durch den Sollwert des Blattwinkels $\theta_{c}$ wird durch ein lineares System zweiter Ordnung mit Eigenfrequenz $\omega$ und Dämpfungsfaktor $\xi$ modelliert. Die Gleichungen (1b) und (1c) werden nur für die Simulation und nicht im Entwurf der Vorsteuerung verwendet.

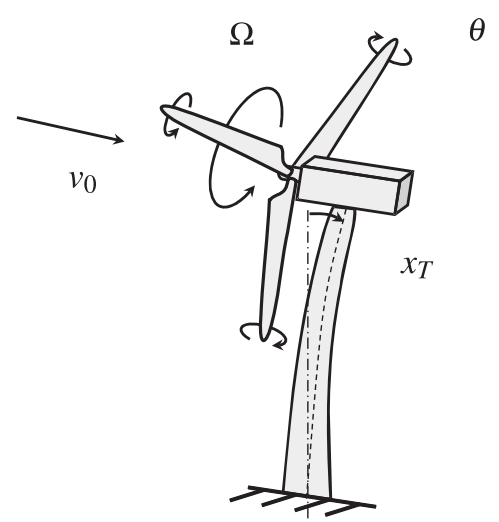

Bild 1 Freiheitsgrade des vereinfachten Modells.
Die Nichtlinearität des Modells konzentriert sich auf die Beschreibung des aerodynamischen Moments $M_{a}$ und des Schubs $F_{a}$ :

$M_{a}=\frac{1}{2} \rho \pi R^{3} \frac{c_{P}(\lambda, \theta)}{\lambda} v_{r e l}^{2}$

$F_{a}=\frac{1}{2} \rho \pi R^{2} c_{F}(\lambda, \theta) v_{r e l}^{2}$.

Hier sind $\rho$ die Luftdichte, $R$ der Rotorradius, $c_{P}(\lambda, \theta)$ und $c_{F}(\lambda, \theta)$ als Tabellen hinterlegten Leistungs- und Schubbeiwerte. Die Schnelllaufzahl

$\lambda=\frac{\Omega R}{v_{r e l}}$

definiert das Verhältnis der Blattspitzengeschwindigkeit zur relativen Anströmung

$v_{r e l}=v_{0}-\dot{x}_{T}$

zwischen der rotoreffektiven Windgeschwindigkeit $v_{0}$ und der Turmkopfgeschwindigkeit $\dot{x}_{T}$, um die aerodynamische Dämpfung abzubilden. Mit der Nabenhöhe $h_{H}$ kann schließlich das Turmfußbiegemoment $M_{y T}$ berechnet werden:

$M_{y T}=h_{H}\left(c_{T} \dot{x}_{T}+k_{T} x_{T}\right)$.

Dieses reduzierte Modell kann im Gegensatz zu den normalerweise für Simulationen verwendeten, aeroelastischen Modellen für den nichtlinearen Regler- und Beobachterentwurf verwendet werden.

\section{Reglerentwurf}

Die Regelung von Windenergieanlagen wird in einen Teillast- und einen Volllastbereich eingeteilt [10]. Im Teillastbereich für niedrige Windgeschwindigkeiten ist es Aufgabe des Reglers, aerodynamische Optimalität zu erreichen. Im Gegensatz dazu sollen im Volllastbereich die Belastungen für die Anlage reduziert werden. Diese Arbeit beschränkt sich auf den Volllastbereich. In diesem Kapitel wird zunächst der Standardregler und dann der Entwurf der Vorsteuerung beschrieben.

\subsection{Standardregler}

In dieser Arbeit wird der Regler von [11] verwendet: Im Volllastbereich von $11,3 \mathrm{~m} / \mathrm{s}$ bis $25 \mathrm{~m} / \mathrm{s}$ wird die Drehzahl $\Omega$ der Windenergieanlage $\Sigma$ bei einer Änderung der rotoreffektiven Windgeschwindigkeit $v_{0}$ durch den Sollwert des kollektiven Blattwinkels $\theta_{c}$ auf die Nenndrehzahl $\Omega_{\text {rated }}=12,1 \mathrm{U} /$ min geregelt, siehe Bild 2. Der Regler $\Sigma_{\mathrm{FB}}$ besteht hier aus einem Tiefpassfilter, um Resonanzen in hohen Frequenzen zu vermeiden, aus einem PI-Regler und einer vom Blattwinkel $\theta$ abhängigen Verstärkung ("Gain Scheduling"), um die unterschiedlichen Steigungen in den $c_{P}$-Kurven auszugleichen. Die Reglerparameter sind so eingestellt, dass ein Kompromiss zwischen der Reduktion der Drehzahlschwankungen, der Stellaktivität und den Belastungen der Anlage erreicht wird. Um Leis- 


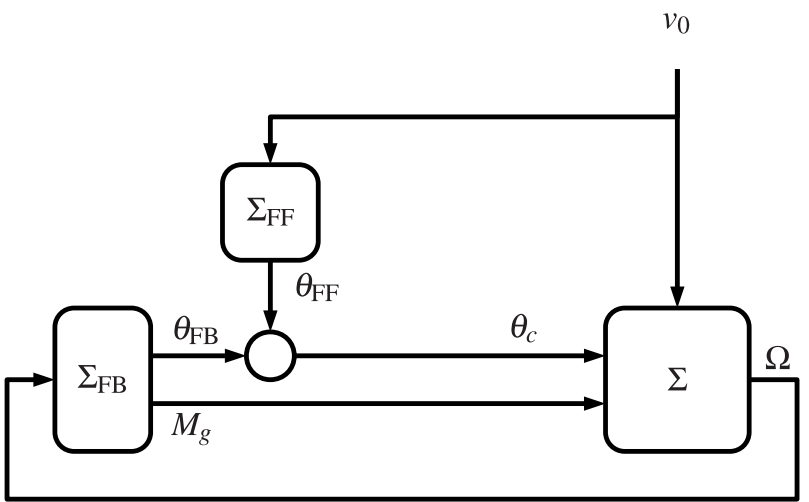

Bild 2 Regelkreis für den Volllastbereich mit Vorsteuerung.

tungsschwankungen zu minimieren, wird zusätzlich das Generatormoment entsprechend der aktuellen gefilterten Drehzahl geregelt.

\subsection{Entwurf Vorsteuerung}

Die Vorsteuerung basiert auf [5]: Ist die rotoreffektive Windgeschwindigkeit $v_{0}$ messbar und vernachlässigt man die Turmbewegung, dann kann der Einfluss des Windes auf die Drehzahl exakt kompensiert werden. Dazu muss der Blattwinkel $\theta$ entlang der stationären Kurve $\theta_{s s}\left(v_{0 s s}\right)$ der Windgeschwindigkeit nachgeführt werden, um das aerodynamische Moment (2a) konstant zu halten. Die stationäre Kurve des Blattwinkels lässt sich aus der stationären Betrachtung von (1), aber auch aus Messdaten gewinnen.

Die Verzögerung durch die Blattverstellung (1c) kann durch eine Inversion der Dynamik berücksichtigt werden. Im Gegensatz dazu ist eine vom dynamischen Verhalten der Blattverstellung unabhängige Vorsteuerung $\Sigma_{\mathrm{FF}}$

$\theta_{\mathrm{FF}}(t)=\theta_{s s}\left(v_{0}(t+\tau)\right)$,

wobei die Prädiktionszeit $\tau$ im nominellen Fall die Verzögerungszeit der Blattverstellung, beim realen Einsatz auch zusätzliche Verzögerungen kompensiert.

Für den realen Einsatz ist es von Vorteil, wenn die Vorsteuerung im laufenden Betrieb ohne Einschwingen des Regelkreises hinzu- oder abgeschaltet werden kann. Dies wird durch die Verwendung der Ableitung

$\dot{\theta}_{\mathrm{FF}}(t)=\dot{v}_{0}(t+\tau) \frac{d \theta_{s s}}{d v_{0 s s}}\left(v_{0}(t+\tau)\right)$

realisiert, welche auf den Integrator des PI-Reglers gegeben werden kann.

Vorteile dieser nichtlinearen Vorsteuerung sind, dass im Gegensatz zu komplexeren Ansätzen [2;6] nur grundlegende, einfach zu gewinnende Modellkenntnisse benötigt werden.

\section{Ergebnisse bei perfekten Messungen}

Zunächst soll gezeigt werden, welche Verbesserungen durch die Vorsteuerung erzielt werden können, wenn

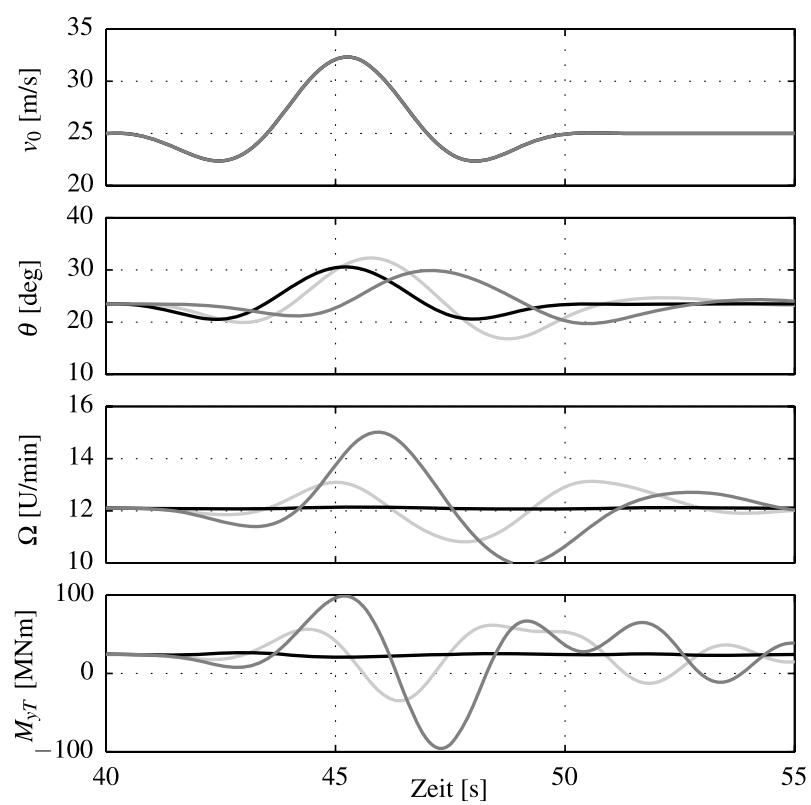

Bild 3 Simulationsergebnisse bei perfekter Messung mit einem aeroelastischen Modell: Ohne Vorsteuerung (dunkelgrau), prädiktive Vorsteuerung durch ein LIDAR-System (schwarz) und verzögerte Vorsteuerung durch ein Anemometer (hellgrau).

die rotoreffektive Windgeschwindigkeit perfekt messbar ist. Dazu wird eine für die Zertifizierung von Anlagen verwendete extreme Böe (siehe Bild 3 oben) sowohl für die Vorsteuerung (7) verwendet, als auch als Störung auf ein aeroelastisches Modell [11] gegeben, welches um eine kollektive Blattverstellung (1c) erweitert wurde und damit insgesamt 34 dynamische Zustände besitzt. Weiterhin wird angenommen, dass ein perfekt messendes LIDAR-System die Windgeschwindigkeit mit einer Prädiktionszeit $\tau=0,2 \mathrm{~s}$ vorhersagen kann, um die Verzögerungszeit der Blattverstellung zu kompensieren. Mithilfe von "Taylor's Frozen Turbulence" Hypothese [12] kann angenommen werden, dass sich Turbulenzen mit der mittleren Windgeschwindigkeit $\bar{u}$ auf die Anlage zu bewegen. Deshalb müsste das LIDARSystem bei $\bar{u}=25 \mathrm{~m} / \mathrm{s}$ mindestens $5 \mathrm{~m}$ vor der Anlage messen. Ein perfekt messendes Anemometer, welches sich $5 \mathrm{~m}$ hinter der Rotorfläche auf der Gondel befindet, könnte $v_{0}$ nur mit einer Verspätung $\tau=-0,2 \mathrm{~s}$ erfassen. Bild 3 zeigt, dass mit beiden Windmessungen Verbesserungen gegenüber dem Fall ohne Vorsteuerung erzielt werden können. Die maximale Drehzahländerung wird durch die prädiktive Vorsteuerung von 2,92 U/min auf $0,0498 \mathrm{U} / \mathrm{min}$ reduziert. Eine perfekte Kompensation ist nicht möglich, da beim Entwurf Zustände vernachlässigt wurden, wie z. B. die Turmschwingung. Trotzdem kann mit der Drehzahländerung auch das maximale Turmfußbiegemoment von $98,8 \mathrm{MNm}$ auf $26,6 \mathrm{MNm}$ reduziert werden.

Mit der leicht verspäteten Vorsteuerung wird noch eine Reduktion der maximalen Drehzahländerung auf 1,03 U/min und des maximalen Turmfußbiegemoments auf 61,7 MNm erreicht. Da auf jeder Anlage Anemometer 
zur Betriebsführung installiert sind, könnte diese Verbesserung ohne die Kosten für ein LIDAR-System erreicht werden.

Diese Ergebnisse zeigen, dass die durch einfache mechanische und systemtheoretische Betrachtung gewonnene nichtlineare Vorsteuerung aus regelungstechnischer Sicht auch mit dem komplexen aeroelastischen Modell sehr gute Resultate erzielt und im Gegensatz zu den komplexen, aus linearisierten Modellen gewonnenen Ansätzen (z. B. [2;6]) nicht nur in einem Arbeitspunkt, sondern über den gesamten Volllastbereich gültig ist. Des Weiteren kann gezeigt werden, dass theoretisch nur eine geringe Prädiktionszeit notwendig ist, um die Auswirkung des rotoreffektiven Windes auf die Drehzahl zu kompensieren.

Im Folgenden soll untersucht werden, inwiefern diese Verbesserungen unter realen Messbedingungen noch erreichbar sind.

\section{Windmessungen}

Das Windfeld vor einer Windenergieanlage stellt durch die sich ändernde Turbulenz eine komplexe Störgröße dar. In diesem Kapitel werden Möglichkeiten untersucht, um den einströmenden Wind einer Windenergieanlage zu messen. Die Daten entstammen einer Messkampagne, welche von Oktober 2009 bis Februar 2010 am Prototyp einer AREVA Anlage (Bild 4) in Bremerhaven durchgeführt wurde [13].

\subsection{Windmessungen mit Windenergieanlagen}

Um zu überprüfen, inwiefern es möglich ist, mit Ultraschall und LIDAR die rotoreffektive Windgeschwindigkeit zu erfassen, wird zunächst eine Referenz definiert. Diese berechnet sich mithilfe der Messdaten von Rotordrehzahl, Blattwinkel, Generatormoment und Luftdichte sowie mit dem reduzierten nichtlinearen Modell (1)-(3).

Mit (1a) erhält man zunächst das aerodynamische Moment $M_{a}$. Unter Vernachlässigung der Turmbewegung

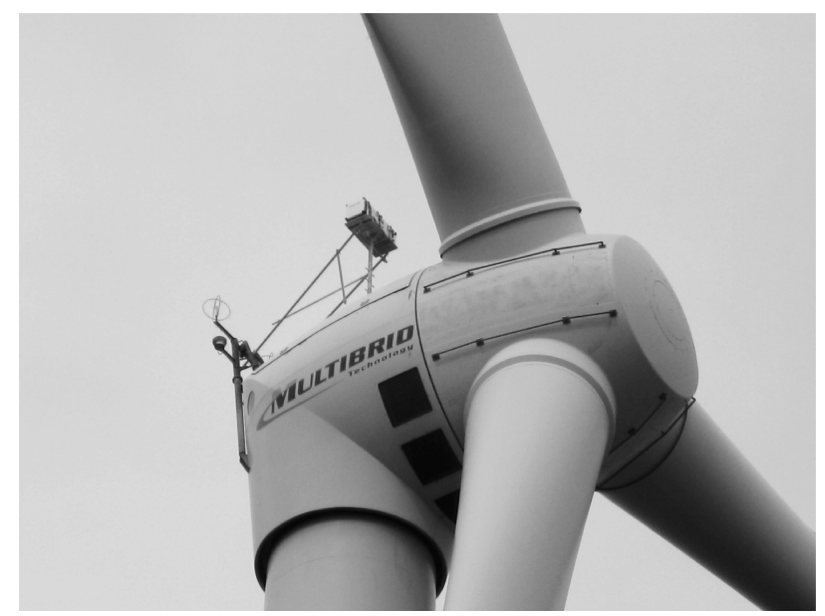

Bild 4 Ultraschallanemometer und SWE-LIDAR auf der Gondel des Prototyps AREVA M5000.

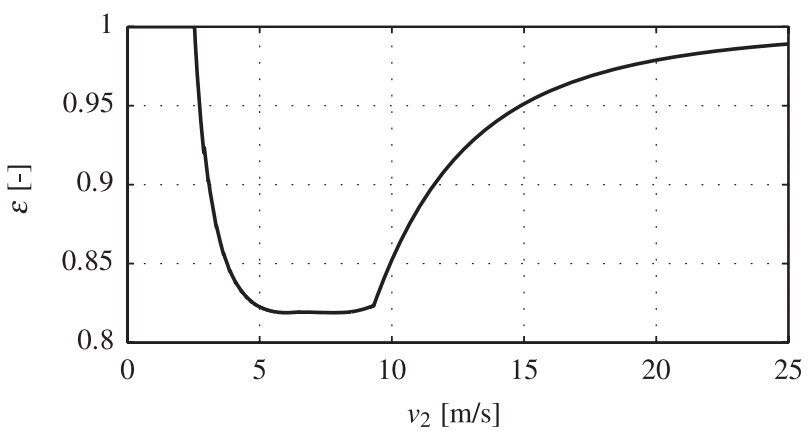

Bild 5 Korrekturfunktion $\epsilon\left(v_{2}\right)$ für die AREVA M5000, um eine Windgeschwindigkeit $v_{2}$ in Rotorebene auf eine unbeeinflusste vor der Anlage $\mathrm{zu}$ korrigieren.

$\left(\dot{x}_{T}=0 \mathrm{~m} / \mathrm{s}\right)$ ergibt sich mit (2a) und (3) eine kubische Gleichung in der unbekannten Schnelllaufzahl $\lambda$ :

$\lambda^{3}=\frac{1}{2} \rho \pi R^{5} \frac{\mathcal{c}_{P}(\lambda, \theta)}{M_{a}} \Omega^{2}$.

Da der Leistungsbeiwert $c_{P}$ von $\lambda$ abhängig ist, kann keine explizite Lösung gefunden werden. Deshalb wurde (8) in diskreten Punkten vorab gelöst. Durch die Lage der Lösungen auf den $c_{P}$-Kurven kann aus den drei mathematischen Lösungen die aerodynamisch richtige identifiziert und eine dreidimensionale Tabelle $v_{0}\left(M_{a}, \Omega, \theta\right)$ erstellt werden. Durch Interpolation kann so aus den Turbinenmessdaten ein Verlauf der geschätzten rotoreffektiven Windgeschwindigkeit $v_{0 R}$ erstellt werden. Vorab werden die Turbinenmessdaten noch mit Kerbfiltern gefiltert, um Resonanzen von Moden, die im reduzierten Modell nicht enthalten sind, nicht dem Wind zuzuschreiben.

Bild 6 zeigt exemplarisch den Verlauf über $30 \mathrm{~min}$.

\subsection{Windmessungen mit Ultraschall}

Der einströmende Wind wird traditionell mit einem Anemometer auf der Gondel einer Windenergieanlage gemessen. Damit wird die Windnachführung realisiert, sowie die Ein- und Ausschaltwindgeschwindigkeit gemessen. Diese Windmessung ist im Betrieb jedoch stark durch die Blätter gestört und durch die Energieumsetzung herrscht hier eine geringere Windgeschwindigkeit. Für die Störgrößenaufschaltung ist der unbeeinflusste Wind $\mathrm{zu}$ bestimmen. Dazu wird im Folgenden eine Korrekturfunktion für Messungen in der Rotorebene hergeleitet: Die aerodynamische Leistung des Windes lässt sich zum einen durch den Leistungsbeiwert und der unbeeinflussten homogenen Windströmung $v_{1}$ vor der Anlage bestimmen [12]:

$P_{a}=\frac{1}{2} \rho \pi R^{2} c_{P} v_{1}^{3}$

Zum anderen wird angenommen, dass der Luftmassenstrom $\dot{m}$ durch die Rotorfläche $\pi R^{2}$ hinter der Anlage auf 


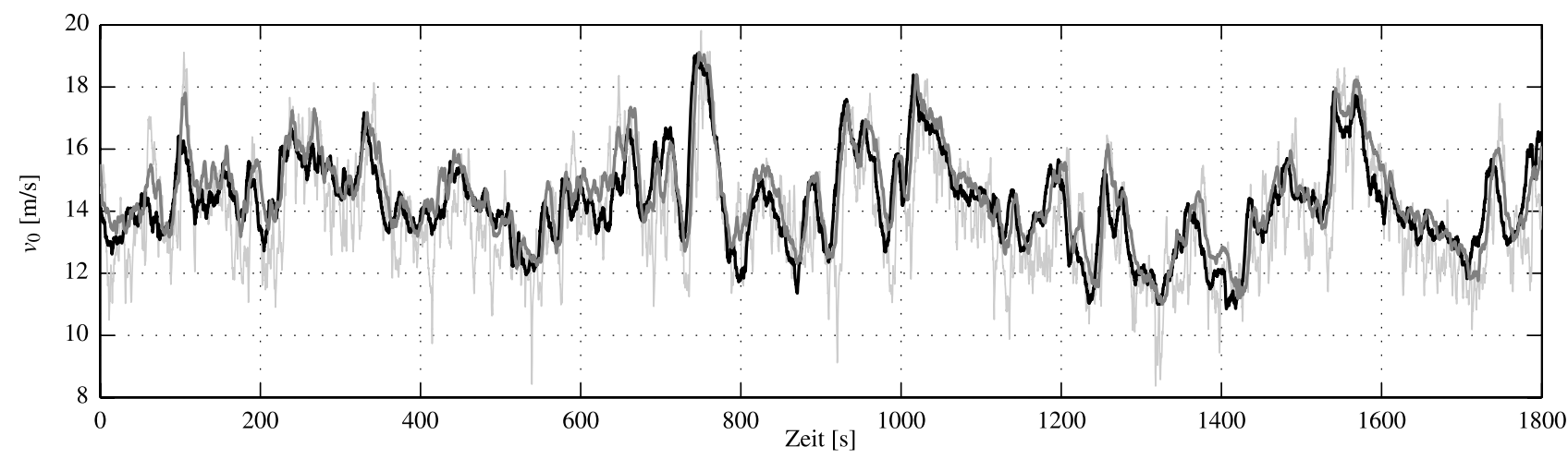

Bild 6 Vergleich der Messdaten für die rotoreffektive Windgeschwindigkeit: Korrigierte Messung Ultraschallanemometer $v_{0 S}$ (hellgrau), Messungen LIDAR $v_{0 L}$ (dunkelgrau) und Schätzung über die Windenergieanlage $v_{0 R}$ (schwarz).

$v_{3}$ abgebremst wird. Die dem Wind entnommene Leistung ist hiermit

$P_{a}=\frac{1}{2} \underbrace{\rho \pi R^{2} v_{2}}_{m}\left(v_{1}^{2}-v_{3}^{2}\right)$.

Nach dem Froude-Rankineschem Theorem [12] gilt für die Windgeschwindigkeit in der Rotorebene

$v_{2}=\frac{1}{2}\left(v_{1}+v_{3}\right)$.

Durch Gleichsetzen von (9) und (10) erhält man unter Verwendung von (11) folgende kubische Gleichung für das Verhältnis $\epsilon=v_{2} / v_{1}$ :

$4 \epsilon^{3}-4 \epsilon^{2}+c_{P}=0$

Nun ist unter Berücksichtigung der Regelung $c_{P}$ stationär nur von $v_{1}$ abhängig. Damit kann $\epsilon$ über den stationären Wert von $v_{1}$ und somit auch über $v_{2}=\epsilon v_{1}$ aufgetragen werden (siehe Bild 5). So können Messdaten eines nahe der Rotorebene messenden Ultraschallanemometers korrigiert werden. Dieses erfasst einen dreidimensionalen Windvektor, wobei mit $u_{S}$ die longitudinale, auf die Rotorebene gerichtete Windkomponente bezeichnet wird. Mit der Annahme, dass nur der frontal auf die Anlage gerichtete Wind zur Energiegewinnung beiträgt, ergibt sich für das Ultraschallanemometers die rotoreffektive Windgeschwindigkeit

$v_{0 S}=u_{S} / \epsilon\left(u_{S}\right)$.

Dieser korrigierte Wert entspricht im Mittel der aus den Turbinendaten geschätzten rotoreffektiven Windgeschwindigkeit $v_{0 R}$, enthält aber mehr hochfrequente Anteile (siehe Bild 6).

\subsection{Windmessungen mit LIDAR}

Im Gegensatz zum Ultraschallanemometer ermöglicht die LIDAR Technologie Messungen vor einer Windenergieanlage und über die gesamte Rotorfläche. Hierbei wird mit einem infraroten Laserstrahl die Geschwindigkeit von Aerosolen in Strahlrichtung ("line-of-sight") über den optischen Dopplereffekt gemessen. Am SWE wurde

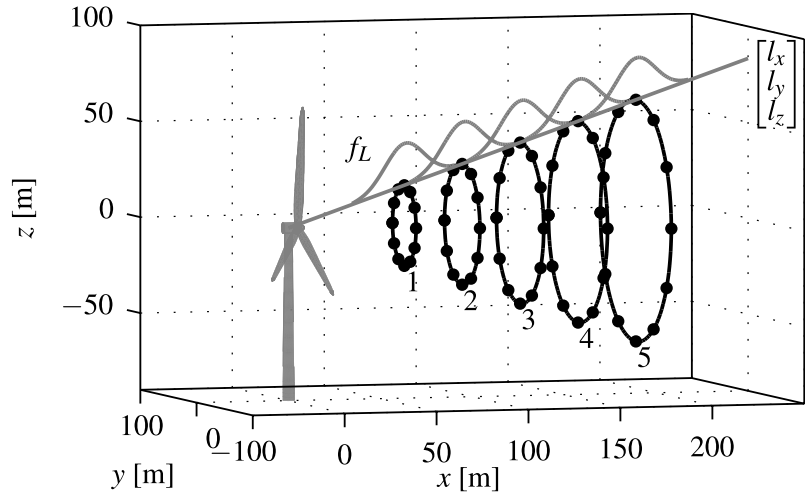

Bild 7 Räumliche Darstellung der Messpositionen und Mittlungen für die verwendete Trajektorie und Pulslänge.

ein kommerzielles, gepulstes LIDAR-System durch eine Scannereinheit erweitert. Diese lenkt mithilfe eines frei beweglichen Spiegels den Laserstrahl in zuvor geplante Positionen [13].

In dem betrachteten Messzeitraum wurden innerhalb von $T_{\text {Scan }}=2,4 \mathrm{~s}$ je zwölf Fokuspunkte auf einer Kreisbahn in fünf Messentfernungen gleichzeitig abgetastet (Bild 7). Durch die Pulslänge und die Datenverarbeitung wird aber nicht nur in den Punkten selbst gemessen, sondern in einem Volumen entlang des Laserstrahles. Mit der als Normalverteilung mit Halbwertsbreite von $30 \mathrm{~m}$ angenäherte Gewichtungsfunktion $f_{L}$ kann die vom LIDAR erfasste Windkomponente $v_{l o s, i j}$ wie folgt modelliert werden:

$v_{l o s, i j}=\int_{-\infty}^{\infty}\left(l_{x} u(a)+l_{y} v(a)+l_{z} w(a)\right) f_{L}(a) d a$,

wobei $a$ die Entfernung zum Fokuspunkt $\left[x_{i j} y_{i j} z_{i j}\right]^{T}$, sowie $u, v$ und $w$ die Windkomponenten in $x, y$ und $z$ Richtung bezeichnen. $\left[\begin{array}{lll}l_{x} & l_{y} & l_{z}\end{array}\right]^{T}$ ist der normierte Vektor vom LIDAR System zum Fokuspunkt. Unter Vernachlässigung der $v$ - und $w$-Komponenten wird zunächst über die jeweils letzten zwölf Messungen einer Messebene gemittelt:

$v_{0 L j}=\frac{1}{12} \sum_{i=1}^{12} \frac{v_{l o s, i j}}{l_{x i}}$. 


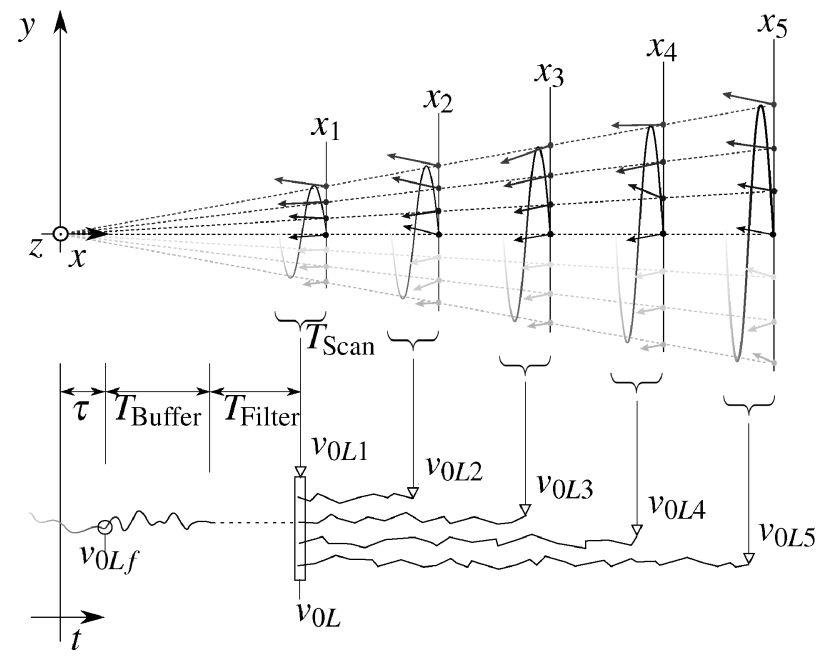

Bild 8 Zusammenhang räumlicher Messung und zeitlicher Verarbeitung: Messungen in den Entfernungen $x_{j}$ werden $\mathrm{zu}$ einer rotoreffektiven Windgeschwindigkeit $v_{0 L}$ kombiniert, gefiltert und so verzögert, dass die Vorsteuerung rechtzeitig reagiert.

Taylors Hypothese nimmt an, dass sich der Wind mit der mittleren Windgeschwindigkeit $\bar{u}$ fortbewegt. Damit ergibt sich ein räumlich-zeitlicher Zusammenhang der Messungen (Bild 8) und die Messebenen können zu der rotoreffektiven Windgeschwindigkeit kombiniert werden:

$v_{0 L}(t)=\frac{1}{5} \sum_{j=1}^{5} v_{0 L j}\left(t-\frac{x_{j}-x_{1}}{\bar{u}}\right)$.

In der beschriebenen Messkampagne wurden die Windmessungen noch nicht für die Regelung verwendet. Sie bieten jedoch die Möglichkeit, in Simulationen abzuschätzen, welchen Effekt eine Vorsteuerung gehabt hätte.

\section{Entwurf einer adaptiven Vorsteuerung}

Die Betrachtung der Windmessungen haben gezeigt, dass die Reaktion der Turbine mit Ultraschall und LIDAR nicht exakt vorhergesagt werden kann: Die rotoreffektiven Windgeschwindigkeiten stimmen in niederen Frequenzen überein, hochfrequent zeigen sich jedoch Unterschiede. In diesem Kapitel wird eine Modifikation der Vorsteuerung beschrieben, die dies adaptiv berücksichtigt.

\subsection{Erweiterung der Modellierung}

Um die Effekte bei der Messung in der Vorsteuerung zu beachten, ist eine Erweiterung der Modellierung nötig, siehe Bild 9. Mit dieser Modellvorstellung wirkt einerseits das einströmende Windfeld $\mathcal{V}$ durch die Evolution $\Sigma_{\mathrm{E}}$ auf dem Weg zum Rotor als Störung $v_{0}$ auf die Anlage. Andererseits wird $\mathcal{V}$ durch die Messung $\Sigma_{\mathrm{M}}$ als $v_{0 M}$ erfasst. Wäre die Übertragung $\Sigma_{\mathrm{E}} \Sigma_{\mathrm{M}}^{-1}$ bekannt, könnte aus der Messung $v_{0 M}$ die rotoreffektive Windgeschwindigkeit $v_{0}$ perfekt rekonstruiert und in der Vorsteuerung (7) verwendet werden.

Eine exakte Modellierung der Windevolution und eine Invertierung der Messung erscheint durch das Messprin- zip unmöglich. Obwohl keine realen Signale übertragen werden, erleichtert die Betrachtung als Übertragungsfunktion die Abschätzung von $\Sigma_{\mathrm{E}} \Sigma_{\mathrm{M}}^{-1}$.

Für die Übertragungszeit kann Taylors Hypothese verwendet werden. Die Amplitude der Übertragung kann aus dem Autospektrum $S_{M M}$ des Signals $v_{0 M}$ und dem Kreuzspektrum $S_{M R}$ zwischen $v_{0 M}$ und $v_{0}$ abgeschätzt werden [14]:

$\left|G_{M R}\right|=\left|\frac{S_{M R}}{S_{M M}}\right|$.

Diese Spektren können aus den spektralen Eigenschaften des Windes gewonnen werden [15]. Hier wird exemplarisch (17) für das Ultraschallanemometer modelliert $(M \rightarrow S)$. Durch die Korrektur (13) kann angenommen werden, dass das Autospektrum $S_{S S}$ einem KaimalWindspektrum $S_{u u}(k)$ entspricht, welches von der Wellenzahl $k=2 \pi f / \bar{u}$ abhängig ist [12]. Des Weiteren kann die rotoreffektive Windgeschwindigkeit als Summe von $n$ Windkomponenten in $x$-Richtung modelliert werden:

$v_{0}=\frac{1}{n} \sum_{i=1}^{n} u_{i}$.

Mithilfe der Eigenschaften der Fouriertransformation (Normierungen werden weggelassen) kann das Kreuzspektrum berechnet werden:

$$
\begin{aligned}
S_{S R} & =\mathcal{F}\left(u_{S}\right) \mathcal{F}^{*}\left(v_{0}\right) \\
& =\mathcal{F}\left(u_{S}\right) \frac{1}{n} \sum_{i=1}^{n} \mathcal{F}^{*}\left(u_{i}\right) \\
& =\frac{S_{u u}(k)}{n} \sum_{i=1}^{n} \gamma\left(r_{S i}, k\right),
\end{aligned}
$$

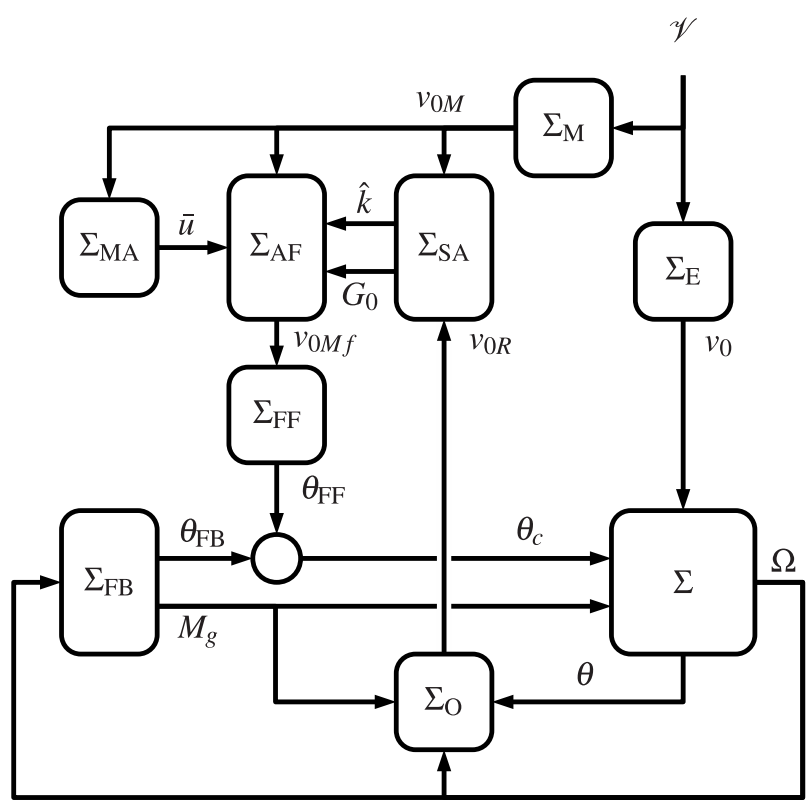

Bild 9 Erweiterter Regelkreis für die kollektive Blattverstellung mit adaptiver Vorsteuerung und Störgrößenbeobachter. 

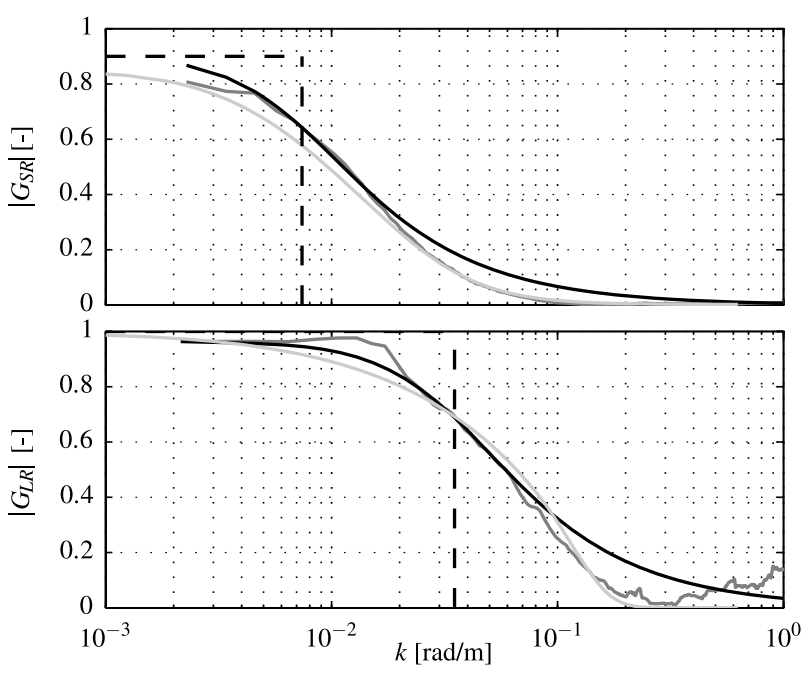

Bild 10 Übertragungsfunktion des Ultraschallanemometers (oben) und des LIDAR Systems (unten) auf den Rotor: gemessen (dunkelgrau), modelliert (hellgrau) und angenähert (schwarz).

wobei $\gamma\left(r_{S i}, k\right)$ die Kohärenz bezeichnet, welche neben $k$ auch vom Abstand $r_{S i}$ des $i$-ten Punktes auf dem Rotor zum Anemometer abhängig ist [12]. Bild 10 zeigt den Verlauf der modellierten Übertragungsfunktion. Ein Vergleich mit der Berechnung von (17) aus den Messdaten zeigt eine gute Übereinstimmung. Da $v_{0}$ nicht bekannt ist, wird dazu der geschätzte Wert $v_{0 R}$ verwendet.

Dies verdeutlicht, dass nicht so sehr die Störung auf der Gondel für die geringe Korrelation verantwortlich ist, als vielmehr die Beschränkung auf einen Punkt: Durch die spektralen Eigenschaften des Windes ist die Korrelation eines Punktes mit der gesamten Rotorfläche nur in der hier modellierten Form zu erwarten.

Auch für die LIDAR-Messung $(M \rightarrow L)$ kann (17) mit (14)-(16) entsprechend berechnet werden, wobei hier die zeitliche Abfolge und die Windevolution beachtet werden müssen [15]. Bild 10 zeigt eine gute Übereinstimmung mit den Messdaten.

\subsection{Adaptiver Filterentwurf}

Die oben modellierte Übertragung ist von der mittleren Windgeschwindigkeit $\bar{u}$ abhängig. Um ein Signal möglichst ähnlich $\mathrm{zu} v_{0}$ aus der Messung $v_{0 M} \mathrm{zu}$ erhalten, wird ein adaptiver Filter entworfen, bestehend aus einem linearen Tiefpassfilter mit variablen Parametern und einer Verzögerung:

$\Sigma_{\mathrm{AF}}=G_{\text {Filter }} e^{-T_{\mathrm{Buffer}} s} \approx \Sigma_{\mathrm{E}} \Sigma_{\mathrm{M}}^{-1}$.

Der Filter $G_{\text {Filter }}$ wird durch Annäherung im Amplitudengang an den Verlauf der Übertragungsfunktion $G_{M R}$ entworfen, da diese nicht direkt verwendet werden kann. Hier wird ein Tiefpass erster Ordnung mit Verstärkung $G_{0}$ und mit folgender Eckfrequenz gewählt:

$f_{\text {cutoff }}=\frac{\hat{k} \bar{u}}{2 \pi}$, wobei $\hat{k}$ die maximale kohärente Wellenzahl ist, bei der $G_{M R}$ auf $-3 \mathrm{~dB}$ unter der stationären Verstärkung $G_{0}$ gefallen ist. Damit ergibt sich als Übertragungsfunktion

$G_{\text {Filter }}=G_{0} \frac{\hat{k} \bar{u}}{s+\hat{k} \bar{u}}$.

Dies ist als diskreter Filter implementiert, dessen Filterparameter zur Laufzeit kontinuierlich mit Änderung von $\bar{u}$ angepasst werden.

Die Berechnung der Verzögerungszeit $T_{\text {Buffer }}$ ergibt sich für das LIDAR-System aus folgender Überlegung (siehe Bild 8): Der Wind benötigt vom Messort $x_{1}$ zum Rotor die Zeit $T_{\text {Taylor }}=x_{1} / \bar{u}$. Durch die Mittelung über $T_{\text {Scan }}$ wird $v_{0 L}$ um $T_{\text {Scan }} / 2$ verzögert. Die Phasenverschiebung durch den Filter $G_{\text {Filter }}$ wird mit $T_{\text {Filter }}$ angenähert. Um zu erreichen, dass das gefilterte Signal um die Zeit $\tau$ vor dem Auftreffen des Windes auf den Rotor durch die Vorsteuerung aufgeschalten wird, ergibt sich für die Verzögerungszeit

$T_{\text {Buffer }}=T_{\text {Taylor }}-\frac{1}{2} T_{\text {Scan }}-T_{\text {Filter }}-\tau$.

Eine Verzögerungszeit wird für das Anemometer nicht benötigt, da die Messung hinter dem Rotor erfolgt.

Für den adaptiven Filter $\Sigma_{\mathrm{AF}}$ muss somit $\bar{u}$ zur Laufzeit ermittelt werden, siehe Bild 9, da im Betrieb sowohl $f_{\text {cutoff, }}$, als auch $T_{\text {Buffer }}$ angepasst werden müssen.

Die mittlere Windgeschwindigkeit $\bar{u}$ kann dabei durch einen einfachen gleitenden Mittelwert $\Sigma_{\mathrm{MA}}$ bestimmt werden. Die Schwierigkeit liegt hier in der Wahl der Mittelungszeit. Weitere Untersuchungen sind nötig, um zu bewerten, ob Werte aus den Messdaten selbst verwendet werden können, wie etwa das integrale Zeitmaß der Turbulenz [12].

Die maximale Wellenzahl $\hat{k}$ und die stationäre Verstärkung $G_{0}$ können wie oben beschrieben durch eine Spektralanalyse basierend auf Messdaten bestimmt werden. Da die Korrelation in realen Messungen abhängig ist von vielen Faktoren wie Stabilität der Atmosphäre, Aerosolkonzentration und Messfehlern, ist eine automatisierte Bestimmung zur Laufzeit durch eine Spektralanalyse $\Sigma_{\text {SA }}$ von Vorteil. Dazu muss die geschätzte rotoreffektive Windgeschwindigkeit $v_{0 R}$ auch zur Laufzeit durch einen Beobachter $\Sigma_{0}$ basierend auf Kapitel 5.1 zur Verfügung stehen. In [16] konnte gezeigt werden, dass die Spektralanalyse und Bestimmung von $\hat{k}$ und $G_{0}$ auch auf einem Steuergerät mit $400 \mathrm{~Hz}$ realisiert werden kann, allerdings sehr sensibel gegenüber Einstellungen wie z.B. Anzahl der gemittelten Spektren ist. Die robuste, automatisierte Erfassung der Korrelation bedarf weiterer Untersuchungen. Deshalb soll im Folgenden zunächst die Adaptivität nur auf $\bar{u}$ beschränkt bleiben und gezeigt werden, dass die durch eine Spektralanalyse ermittelten Werte einen optimalen Filter ermöglichen. 


\section{Ergebnisse bei realen Messungen}

Die adaptive Vorsteuerung kann mit den aufbereiteten Messdaten getestet werden. Dazu wird die über die Turbinendaten geschätzte rotoreffektive Windgeschwindigkeit $v_{0 R}$ als Störgröße auf das reduzierte Anlagenmodell (1)(5) und $v_{0 S}$ bzw. $v_{0 L}$ für die Vorsteuerung verwendet. Damit kann unter Berücksichtigung realistischer Messungen und Windevolution abgeschätzt werden, welche Änderungen die Vorsteuerung für den betrachteten Zeitraum bewirkt hätte.

Um die Notwendigkeit eines Filters zu zeigen, wird zunächst $v_{0 S}$ bzw. $v_{0 L}$ ungefiltert auf die Vorsteuerung (7) gegeben. Die Ergebnisse in Tabelle 1 zeigen eine extreme Verschlechterung durch die Vorsteuerung mit dem ungefilterten Signal des Ultraschallanemometers: die Standardabweichung der Rotordrehzahl $\sigma(\Omega)$ kann nicht reduziert werden, sondern wird mehr als verdoppelt. Der Einsatz eines Filters führt zu keiner Verbesserung gegenüber dem Standardregler, da durch den Zeitversatz des Filters das vorgesteuerte Signal nur zu spät aufgeschalten werden kann.

Für die ungefilterte, LIDAR basierte Vorsteuerung wird zur Berechnung der Verzögerung (23) die mittlere Windgeschwindigkeit $\bar{u}$ für die $30 \mathrm{~min}$ a priori berechnet und $\tau=0,2 \mathrm{~s}$ gesetzt. Eine Reduktion der Drehzahlvariation kann hierbei zwar erreicht werden, jedoch bei der Betrachtung im Frequenzbereich (siehe Bild 11) sind Erhöhungen im Spektrum der Blattverstellgeschwindigkeit für Frequenzen über $0,07 \mathrm{~Hz}$ sichtbar. Diese Grenze entspricht dem Wert für die maximale kohärente Wellenzahl $\hat{k}$. Die zusätzliche Stellaktivität führt auch zu einer Erhöhung der schädigungsäquivalenten Lasten (DEL) am Turmfuß.

In einem weiteren Schritt kann nun gezeigt werden, dass bei der LIDAR basierten Vorsteuerung der Filter mit $\hat{k}$ zur maximalen Reduktion der Drehzahlvariation führt. Dazu wurde eine Reihe von Simulationen mit unterschiedlichen $\hat{k}$ und Prädiktionszeiten $\tau$ ausgewertet. Die mittlere Windgeschwindigkeit $\bar{u}$ wurde durch einen gleitenden Mittelwert über 3 min bestimmt, die stationäre Verstärkung $G_{0}$ basierend auf Bild 10 fest gewählt.

Bild 12 oben zeigt, dass zum einen die optimale Filtereinstellung nahe bei $\hat{k}$ und die optimale Prädiktionszeit $\tau$

Tabelle 1 Relative Änderung der Standardabweichungen und Lasten bezogen auf den Standardregler für die Vorsteuerung mit Ultraschallanemometer (S) und LIDAR-System (L).

\begin{tabular}{|c|c|c|c|c|c|c|}
\hline & $\begin{array}{l}T_{i} \\
{[\mathrm{~s}]}\end{array}$ & $\begin{array}{l}\hat{k} \\
{[\mathrm{rad} / \mathrm{m}]}\end{array}$ & $\begin{array}{l}\tau \\
{[\mathrm{s}]}\end{array}$ & $\begin{array}{l}\sigma(\dot{\theta}) \\
{[-]}\end{array}$ & $\begin{array}{l}\sigma(\Omega) \\
{[-]}\end{array}$ & $\begin{array}{l}\operatorname{DEL}\left(M_{y T}\right) \\
{[-]}\end{array}$ \\
\hline \multirow[t]{2}{*}{ S } & 2,33 & - & - & 5,46 & 2,59 & 4,03 \\
\hline & 2,33 & 0,007 & - & 1,21 & 1,24 & 1,10 \\
\hline \multirow[t]{3}{*}{$\mathrm{L}$} & 2,33 & - & 0,2 & 1,34 & 0,67 & 1,12 \\
\hline & 2,33 & 0,032 & 0,8 & 0,89 & 0,58 & 0,88 \\
\hline & 7,00 & 0,032 & 1,8 & 0,73 & 0,78 & 0,82 \\
\hline
\end{tabular}
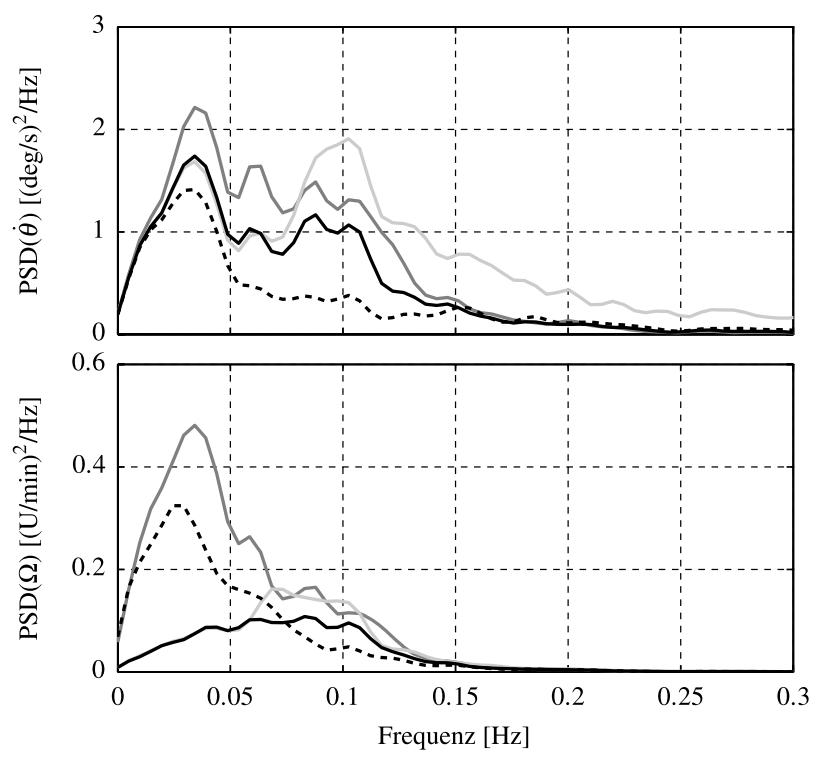

Bild 11 Spektren der Blattverstellgeschwindigkeit und der Rotordrehzahl: Ohne Vorsteuerung (dunkelgrau); mit LIDAR basierter Vorsteuerung: ungefiltert (hellgrau), gefiltert (schwarz), gefiltert und mit modifiziertem Regler (unterbrochen).
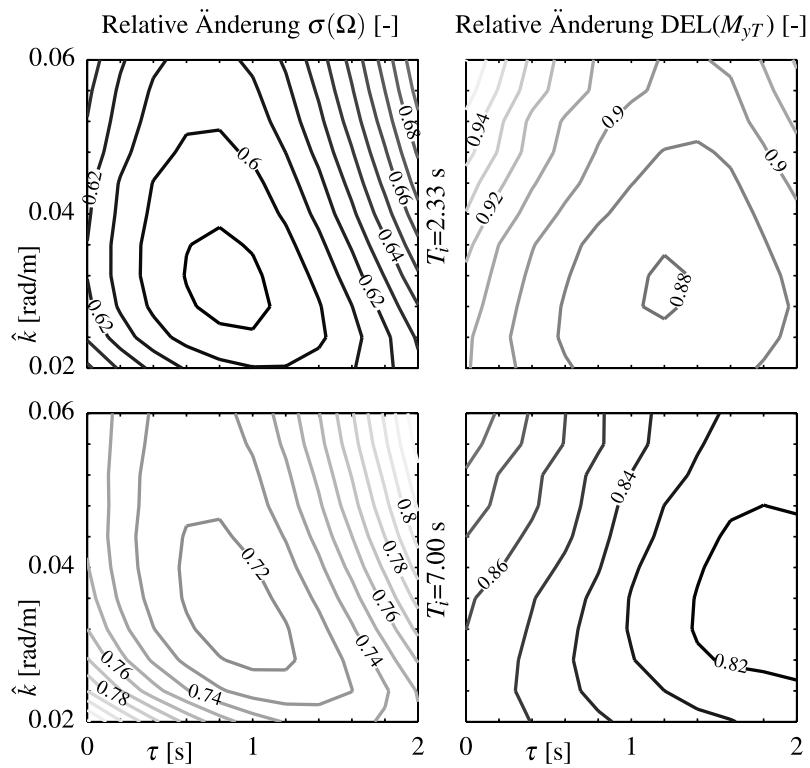

Bild 12 Reduktion der Drehzahlvariation und der Belastung des Turms durch die LIDAR basierte Vorsteuerung mit der ursprünglichen (oben) und einer erhöhten (unten) Integrationszeitkonstante $T_{i}$ des PI-Reglers.

ein wenig höher wie bei den Simulationen mit perfekter Messung liegt. Dies kann durch Verzögerungen in anderen Moden erklärt werden [5]. Zum anderen können durch die Filterung auch die DEL erniedrigt werden. Die Simulationsergebnisse mit den optimalen Einstellungen sind in Bild 11 und Tabelle 1 dargestellt.

Der gewonnene Vorteil in der Standardabweichung der Rotordrehzahl $\sigma(\Omega)$ kann nun genutzt werden, um höhere Reduktionen in der Standardabweichung der Blattverstellgeschwindigkeit $\sigma(\dot{\theta})$ oder den Lasten zu be- 
kommen, indem der PI-Regler modifiziert wird. Werden die Simulationen z. B. mit der dreifachen Integrationszeitkonstante $T_{i}$ wiederholt (Bild 12 unten), können die Lasten am Turmfuß zusätzlich um bis zu $18 \%$ gesenkt werden. Hier ist auch erkennbar, dass das Minimum für $\sigma(\Omega)$ zwar erhöht wird, aber an der gleichen Stelle liegt. Dies kann für die DEL nicht erwartet werden, da die Vorsteuerung mit dem Ziel entworfen wurde, den Effekt auf die Rotordrehzahl zu reduzieren.

Diese Ergebnisse zeigen, dass der Einsatz der Vorsteuerung den Regler von seiner Aufgabe entlastet, die Drehzahl konstant zu halten, und damit die Belastungen auf die Anlage durch einen neu entworfenen Regler weiter reduziert werden können.

\section{Zusammenfassung und Ausblick}

Dieser Beitrag beschreibt eine adaptive Vorsteuerung für die kollektive Blattverstellung bei Windenergieanlagen. Durch die Komplexität des Windes liegt dabei der Schwerpunkt auf der Erfassung der Störgröße. Theoretisch und mit Messdaten kann gezeigt werden, dass nur große Turbulenzen vorhergesagt werden können und ein adaptiver Filter benötigt wird, welcher abhängig von der mittleren Windgeschwindigkeit und der Korrelation der Windmessung mit der Anlage angepasst wird. Durch Simulationen auf Basis von Messdaten kann für die LIDAR basierte Vorsteuerung eine Reduktion der Drehzahlvariation, Belastung und Stellaktivität auch für die Realität prognostiziert werden. Mit einer Anpassung des Feedback-Reglers können die Belastungen weiter reduziert werden.

Im Frühjahr 2012 wurde die Vorsteuerung auf den zwei $600 \mathrm{~kW}$ CART-Testanlagen („Controls Advanced Research Turbine, 2/3-bladed“) des US-amerikanischen Forschungszentrums NREL mit zwei unterschiedlichen LIDAR-Systemen erfolgreich getestet. Das scannende LIDAR System des SWE auf der CART2 erzielte in einer Korrelationsstudie vor der Erprobung eine maximale kohärente Wellenzahl von $0,06 \mathrm{rad} / \mathrm{m}$ [7]. In den Zeiträumen, in denen die zuvor bestimmte maximale kohärente Wellenzahl erreicht wurde, konnte eine Reduktion von circa $30 \%$ in der Standardabweichung der Generatordrehzahl erzielt werden (Bild 13 oben). Da wie in dieser Arbeit zwar die mittlere Windgeschwindigkeit in der Adaption des Filters beachtet wurde, die Wellenzahl jedoch nicht nachgeführt wurde, erhöhte sich in den Abschnitten mit schlechterer Korrelation durch einen Messfehler die Standardabweichung der Generatordrehzahl um circa $30 \%$. Mit einem kommerziellen System mit drei festen Messpositionen auf der CART3 konnte nur eine maximale kohärente Wellenzahl von $0,03 \mathrm{rad} / \mathrm{m}$ erreicht werden [8]. Damit konnte die Standardabweichung der Generatordrehzahl um circa $10 \%$ reduziert werden (Bild 13 unten). Diese Ergebnisse bestätigen, dass mit der vorgeschlagenen Vorsteuerung auch unter realen Bedingungen eine Verbesserung in der Regelung erzielt werden kann, die unterschiedliche Erfassung der rotor-
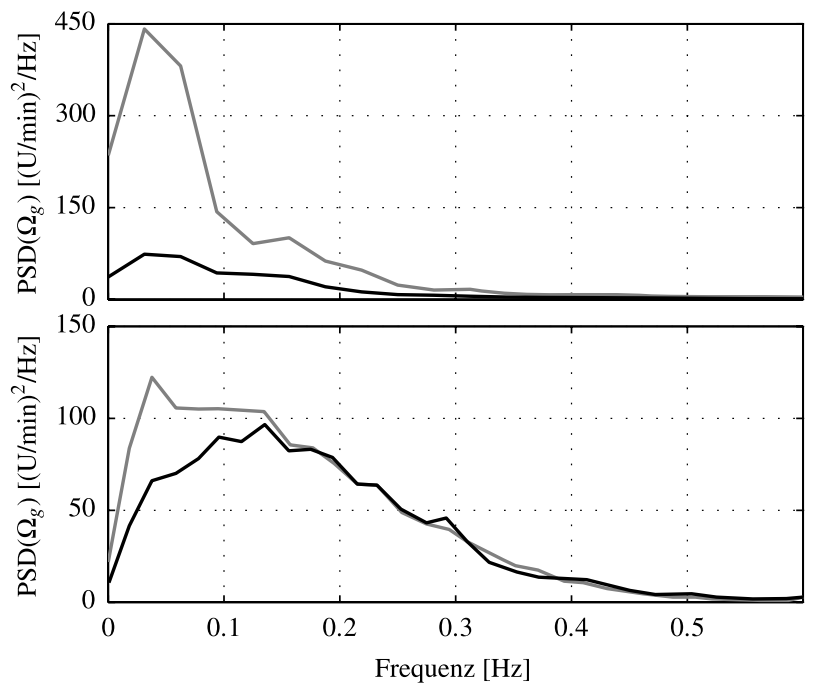

Bild 13 Spektren der Generatordrehzahl von Feldtests auf einer Forschungsanlage mit 2 Rotorblättern (oben, [7]) und mit 3 Rotorblättern (unten, [8]): Ohne Vorsteuerung (dunkelgrau); mit LIDAR basierter adaptiver Vorsteuerung (schwarz).

effektiven Windgeschwindigkeit jedoch beachtet werden muss. Im Rahmen des Forschungsprojekts „LIDAR II“ soll eine Implementierung auf einer $5 \mathrm{MW}$ Offshore Anlage im Testfeld „alpha ventus“ erfolgen.

\section{Danksagung}

Diese Arbeit wurde im Rahmen des Verbundforschungsprojekts "LIDAR II" durch das Bundesministerium für Umwelt, Naturschutz und Reaktorsicherheit gefördert. Vielen Dank an das SWE-LIDAR Team und an Björn Siegmeier von AREVA Wind GmbH für die Unterstützung bei den LIDAR-Messungen.

\section{Literatur}

[1] K. Graichen, V. Hagenmeyer, und M. Zeitz, "A new approach to inversion-based feedforward control design for nonlinear systems," Automatica, vol. 41, pp. 2033-2041, 2005.

[2] F. Dunne, D. Schlipf, L. Y. Pao, A. D. Wright, B. Jonkman, N. Kelley, und E. Simley, "Comparison of two independent lidar-based pitch control designs," in AIAA Aerospace Sciences Meeting Including the New Horizons Forum and Aerospace Exposition, Nashville, USA, 2012.

[3] D. Schlipf, S. Kapp, J. Anger, O. Bischoff, M. Hofsäß, A. Rettenmeier, U. Smolka, und M. Kühn, "Prospects of optimization of energy production by LiDAR assisted control of wind turbines," in EWEA Annual event, Brüssel, Belgien, 2011.

[4] E. A. Bossanyi, A. Kumar, und O. Hugues-Salas, "Wind turbine control applications of turbine-mounted LIDAR," in The Science of Making Torque from Wind, Oldenburg, Deutschland, 2012.

[5] D. Schlipf, T. Fischer, C. E. Carcangiu, M. Rossetti, und E. Bossanyi, "Load analysis of look-ahead collective pitch control using LiDAR," in Deutsche Windenergiekonferenz DEWEK, Bremen, Deutschland, 2010.

[6] N. Wang, K. Johnson, und A. Wright, "FX-RLS-based feedforward control for LIDAR-enabled wind turbine load mitigation," IEEE Transactions on Control Systems Technology, vol. 20, no. 5, pp. 1212-1222, 2012. 
[7] D. Schlipf, P. Fleming, F. Haizmann, A. Scholbrock, M. Hofsäß, A. Wright, und P.W. Cheng, "Field testing of feedforward collective pitch control on the CART2 using a nacelle-based lidar scanner," in The Science of Making Torque from Wind, Oldenburg, Deutschland, 2012.

[8] A. Scholbrock, P. Fleming, L. Fingersh, A. Wright, D. Schlipf, F. Haizmann, und F. Belen, "Field testing LIDAR based feedforward controls on the NREL controls advanced research turbine," in AIAA Aerospace Sciences Meeting Including the New Horizons Forum and Aerospace Exposition, Dallas, USA, 2013.

[9] D. Schlipf, D. J. Schlipf, und M. Kühn, "Nonlinear model predictive control of wind turbines using LIDAR," Wind Energy, 2012.

[10] M. Geyler und P. Caselitz, "Regelung von drehzahlvariablen Windenergieanlagen," at - Automatisierungstechnik, vol. 56, pp. 614-626, 2008.

[11] J. Jonkman, S. Butterfield, W. Musial, und G. Scott, "Definition of a 5-MW reference wind turbine for offshore system development," Technical Report NREL/TP-500-38060, 2009.

[12] T. Burton, N. Jenkins, D. Sharpe, und E. Bossanyi, Wind Energy Handbook. New York, USA: John Wiley \& Sons, 2011.

[13] A. Rettenmeier, O. Bischoff, M. Hofsäß, D. Schlipf, J. J. Trujillo, und M. Kühn, "Wind field analysis using a nacelle-based lidar system," in European Wind Energy Conference, Warschau, Polen, 2010.

[14] J.S. Bendat und A. G. Piersol, Random data; analysis and measurement procedures. New York, USA: John Wiley \& Sons, 1971.

[15] D. Schlipf, J. Mann, und P. W. Cheng, "Model of the correlation between lidar systems and wind turbines for lidar assisted control," in unter Begutachtung beim Journal of Atmospheric and Oceanic Technology, 2013.
[16] F. Haizmann, P. Fleming, D. Schlipf, A. Scholbrock, M. Hofsäß, A. Wright, und P. W. Cheng, "Implementation of an online correlation analysis for an adaptive lidar based feed-forward controller for wind turbines," in EWEA Annual event, Wien, Österreich, 2013.

Manuskripteingang: 8. August 2012

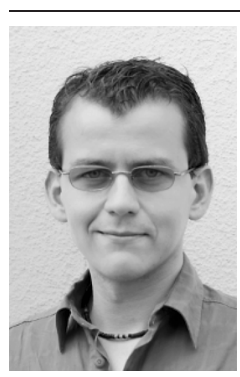

Dipl.-Ing. David Schlipf ist Doktorand des Stiftungslehrstuhls Windenergie der Universität Stuttgart. Hauptarbeitsgebiet: LIDAR basierte Regelung von Windenergieanlagen.

Adresse: Stiftungslehrstuhl Windenergie am Institut für Flugzeugbau, Allmandring 5B, D-70569 Stuttgart, E-Mail: schlipf@ifb.uni-stuttgart.de

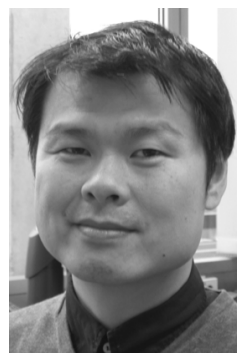

Prof. Dr. Po Wen Cheng ist Leiter des Stiftungslehrstuhls Windenergie der Universität Stuttgart.

Adresse: Stiftungslehrstuhl Windenergie am Institut für Flugzeugbau, Allmandring 5B, D-70569 Stuttgart, E-Mail: cheng@ifb.uni-stuttgart.de 